Open Access

\title{
A ranging code based on the improved Logistic map for future GNSS signals: code design and performance evaluation
}

\author{
Dun Wang ${ }^{1}$, Rui Xue $2^{*}$ and Yanbo Sun ${ }^{2}$
}

\begin{abstract}
Ranging code is the core component of the signal transmission scheme in any global navigation satellite system (GNSS); its performance directly influences on the technical indexes of positioning accuracy, compatibility, interoperability, anti-interference, security, synchronization realization, and so on. Therefore, research on ranging codes could provide theoretical support for the improvement of the performance of ranging codes and extension of their design methods to future satellite navigation signal structures. In order to improve the balance in classical chaotic sequences, a novel ranging code is proposed in this paper and constructed by a series of the improved Logistic-map chaotic sequences with different initial values through weighted optimization, summation, and quantization. Then a comprehensive performance evaluation method based on the Welch bound including three main indexes has been introduced, namely the performance of acquisition, tracking, and robustness against interfering narrowband signals. Finally, the three indexes are combined in a cost function by weighting to evaluate the proposed code, coarse/acquisition (C/A), Gold, Weil, and Random as well as the conventional chaotic codes, and the corresponding weighted coefficients can be adjusted flexibly according to the user groups or application types. Theoretical analysis and simulation results over an additive white Gaussian noise (AWGN) channel show that the proposed ranging code cannot only demonstrate excellent performance in acquisition and anti-narrowband interference while maintaining high quality in tracking performance as the C/A code but also significantly improve balance performance and strengthen reliability and security.
\end{abstract}

Keywords: Satellite navigation, Ranging codes, Chaotic sequences, Weighed processing

\section{Introduction}

The navigation signal is an important part of satellite navigation systems as the coordination work link to satellites, ground control centers, and users [1]. It is directly related to the basic functions of location, time service, and velocity measurement as well as to the key performance indexes of positioning accuracy, compatibility, interoperability, security, anti-interference ability, and so on. The ranging code is the core part of modern satellite navigation signal transmission schemes and has been widely used in Global Navigation Satellite Systems (GNSSs), such as the

*Correspondence: xuerui0216@hotmail.com

${ }^{2}$ College of Information and Communication Engineering, Harbin Engineering University, 150001 Harbin, China

Full list of author information is available at the end of the article
US Global Positioning System (GPS), Russian Global Navigation Satellite System (GLONASS), European Galileo, and Chinese Compass. The properties of ranging code have a great influence on the performance of acquisition, tracking, demodulation, anti-jamming, secrecy, and so on [2]. Therefore, study on properties of ranging codes could provide theoretical support for pursuing ranging codes with better performance and expand design methods of ranging codes in future satellite navigation signal structures.

With the development of GNSSs and regional navigation systems, such as Japanese Quasi-Zenith Satellite System (QZSS) and Indian Regional Navigation Satellite System (IRNSS), the number of navigation signals in space is anticipated over 400 by 2030 [3], which will further aggravate an already crowded radio spectrum in L-band 
(1164 $\sim 1610 \mathrm{MHz})$ and negatively impact the new signal scheme design due to excessive spectrum overlapping [4]. Meanwhile, compatibility and interoperability among different navigation systems are becoming a hot research topic around the world in recent years [5-7]. The isolation of mutual operation signals in various GNSSs completely depends on ranging code, and it plays a decisive role in multiaccess interference degree and compatibility among navigation systems [8].

At present, $M$ code and Gold code generated by linear feedback shift register (LFSR) sequences are the most widely used ranging codes in existing satellite navigation systems, but both of codes have some common disadvantages such as limited number of available code groups and poor anti-decryption ability. With the development of decryption technology to pseudo-noise sequence codes, the above codes are facing the danger of being cracked [9]. In recent 10 years, chaotic sequences have made it possible for ranging as a result of the gradual maturity in theory and application of chaos, which have the following advantages: high sensitivity to initial values, large number of code groups, high linear complexity, and excellent confidentiality. The application of chaotic sequences opens up a new perspective for spread spectrum communication [10], but chaotic sequences also have their shortcomings especially for poor balance. For example, the balance coefficient is larger than 0.02 when sequence length is relatively short, which cannot satisfy the requirement of high-precision positioning systems [11, 12].

The rest of the paper is organized as follows. Section 2 mainly expounds basic principle of the proposed chaotic sequence and other classical chaotic sequences, and analyses their characteristics of balance and correlation. Section 3 provides a weighted performance evaluation method based on the Welch bound. Section 4 introduces three typical interference. Simulation results are discussed in Section 5. Finally, we conclude the paper in Section 6.

\section{Ranging codes}

\subsection{Classical chaotic sequences}

Generally, the one-dimensional discrete chaotic maps can be applied to generate pseudo-random sequences, that is to say a qualified initial value is selected firstly and then a decimal chaotic sequence of length $N$ is formed after $N$ iterations according to chaotic map, for instance, the Chebyshev map, Tent map, Bernoulli map, Logistic map, and improved Logistic map. The details of these maps are shown in Ref. [13-17]. At last, the binary chaotic sequence is obtained through binary quantization. The above mentioned maps are described as follows.

(1) The Chebyshev map

The $q$-order Chebyshev map is denoted as

$$
x_{n+1}=\cos \left(q \times \arccos \left(x_{n}\right)\right)
$$

where initial value $x_{0} \in[-1,1]$. If the positive integer $q$ is equal to any integer powers of 2 , the generated sequence is in the state of chaos. In this case, the binary quantization threshold is 0 .

(2) The Tent map

$$
x_{n+1}=\left\{\begin{array}{cl}
x_{n} / a & \text { if } 0<x_{n}<a \\
\left(1-x_{n}\right) /(1-a) & \text { if } a \leq x_{n} \leq 1
\end{array}\right.
$$

when constant $a \in(0,1)$ and initial value $x_{0} \in[0,1]$, the generated sequence is in the state of chaos. In this case, the binary quantization threshold is 0.5 .

(3) The Bernoulli map

$$
x_{n+1}=\left\{\begin{array}{cl}
x_{n} / b & \text { if } x_{n} \leq b \\
\left(x_{n}-b\right) /(1-b) & \text { if } x_{n}>b
\end{array}\right.
$$

when constant $b \in(0,1)$ and initial value $x_{0} \in[0,1]$, the generated sequence is in the state of chaos. In this case, the binary quantization threshold is 0.5 .

(4) The Logistic map

$$
x_{n+1}=c x_{n}\left(1-x_{n}\right)
$$

where $x_{n} \in(0,1)(n=0,1, \cdots)$, and $0<c \leq 4$ is called the bifurcation parameter, for $c \in(3.5699 \cdots, 4]$ the sequence generated from Eq. (4) is chaotic. In this case, the binary quantization threshold is 0.5 .

(5) The improved Logistic map

The results in Ref. [18] show that the abovementioned maps may not only be used for masking and encoding but are also robust with respect to different types of external interference. Subsequently, Chinese scholars Wang and $\mathrm{Hu}$ put forward to a special Logistic map designated as the improved Logistic map [19] in order to distinguish from the traditional Logistic map. By Setting $x_{n}=\left(z_{n}+1\right) / 2$ and $c=4$, Eq. (4) is changed to the improved Logistic map within symmetrical region as below.

$$
z_{n+1}=1-2 z_{n}^{2}
$$

when $z_{n} \in(-1,1)$, the generated sequence is in the state of chaos, and the binary quantization threshold is 0 . In addition, they derived the mathematical expression of statistical properties of chaotic sequences generated by the improved Logistic map and found that their statistical properties are identical with those of white noise. Thus, the improved Logistic map is more appropriate to be used for spread spectrum (SS) communications than the traditional Logistic map [20].

\subsection{Analysis of balance}

Viewed from information transmission perspective, satellite navigation is a special case of SS communications. In majority of SS communications, one of the most basic requirements for the SS sequence should have excellent balance. There is a close correlation between balance of SS sequence and carrier rejection, and an unbalanced 
sequence will make carrier leakage much bigger, which will easily lead to bit error or information loss [21]. Specifically, on the transmitter side, an unbalance signal will result in leaking some important information of SS sequence, which will make SS signals lost its superiority in concealment and waste emission power simultaneously. For the receiving part, the unbalanced signal as a narrowband interference into the receiver will increase the internal interference of the system and make the improper locking appear at unsubdued carriers, which influences normal work of receiver seriously. Evidently, the study on balance in SS sequences has important significance to authorized users of GNSSs.

In Ref. [19], balance quality is characterized by balance coefficient that is decided by the number of " +1 " and " -1 " (or "0") in a SS sequence. We assume that $A$ and $B$ denote the number of " +1 " and "- 1 " (or " 0 ") in a SS sequence, and balance coefficient $C$ is defined as $C=(A-B) / N$, where $N$ is the length of sequence. It is noteworthy that the nearer $A$ approximates to $B$, the better balance performance we get. Ideally, $C$ is equal to zero when $A$ is the same as $B$. Figure 1 shows the relation curves between balance coefficients of classical chaotic sequences and different code length. As we see from Fig. 1, the fluctuation of balance coefficients in the above five chaotic sequences is violent even greater than 0.02 when code length is varied from 2000 to 5000. Furthermore, a chaotic sequence using the improved Logistic map without any processing has the worst performance in balance because its balance coefficient is always more than 0.06 at various code length. In this paper, the problem can be solved by constructing a new chaotic sequence or optimizing the already existing chaotic sequences according to certain rules.

\subsection{A novel chaotic sequence based on the improved Logistic map with weighted processing}

In order to improve the balance performance of classical chaotic sequences as ranging codes, a novel chaotic sequence based on the improved Logistic map is proposed. Construction process of the sequence is as follows:

a) $L$ different initial values are randomly selected and substituted into the improved Logistic map (Eq. (5)) respectively.

b) $L$ independent real-valued chaotic sequences $\mathbf{S}_{1}, \mathbf{S}_{2}, \cdots, \mathbf{S}_{L}$ with length $N$ are generated after $N$ iterations.

c) The $k^{\text {th }}$ element $g_{k}$ in the weighted sequence $\mathbf{G}$ is calculated by the following equation, i.e.

$$
g_{k}=p_{1} \times s_{k, 1}+p_{2} \times s_{k, 2}+\cdots+p_{L} \times s_{k, L}
$$

where $k=1,2, \cdots, N, s_{k, L}$ is the $k^{\text {th }}$ element in the $L^{\text {th }}$ sequence, and $p_{1}, p_{2}, \cdots, p_{L}$ are weighted parameters and $p_{1}+p_{2}+\cdots+p_{L}=1$.

d) The proposed ranging code is obtained from the sequence $\mathbf{G}$ through binary quantization.

From construction process of the proposed code, we note that the weighted parameters are the key factor which has serious effect on the balance performance, and balance coefficient is equal to or close to zero can be regarded as criterion for weighted parameter selection. Based on this criterion, a selection method for weighted parameters is provided by this paper, then we set an example to illustrate how the method is carried out under computer simulation.

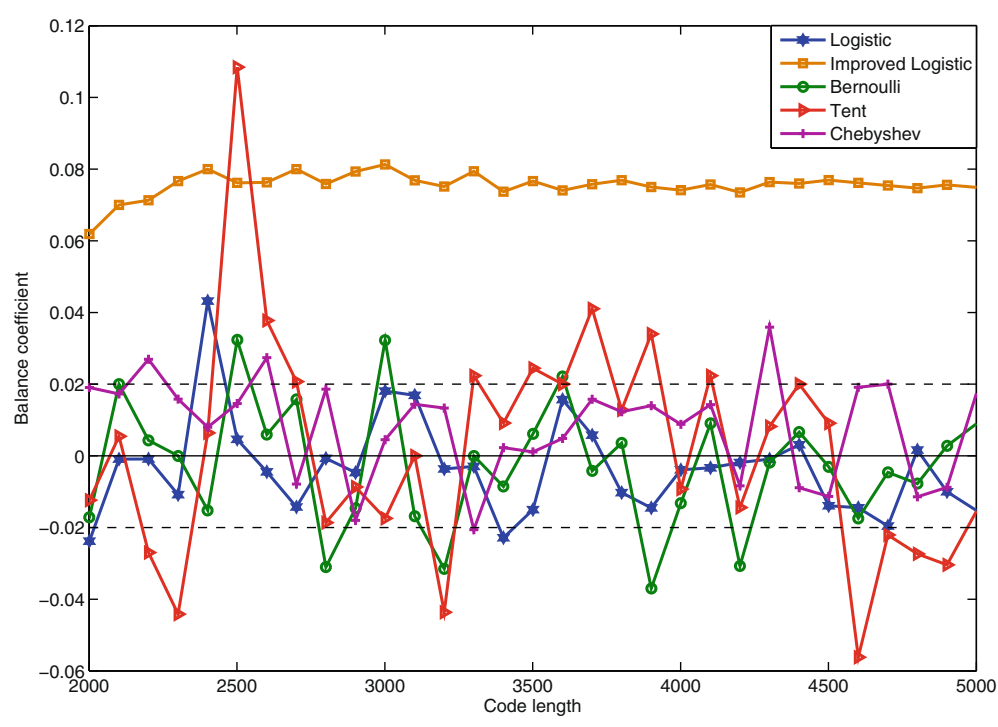

Fig. 1 Relation curves between balance coefficients of classical chaotic sequences and various code length 
Figure 2 shows relation curves between balance coefficients of the proposed sequences and weighted parameters with various code length when $L$ is equal to 2 , where initial values are randomly selected as -0.87 and 0.83 separately. In Fig. 2, we observe that the curves of balance coefficients have certain amount of intersection points with the region of $\mid$ balance coefficient $\mid \leq 5 \times 10^{-5}$ when code length is varied from 256 to 2048. The weighted parameters can be confirmed by drawing perpendicular lines to the horizontal axis "weighted parameter" from these points. For instance, one weighted parameter of the proposed sequence is fixed at 0.49 as shown in Fig. 2 and another is 0.51 when the code length is 2048 .

From Eq. (5), we can deduce that $s_{k, 1}=1-2 s_{k-1,1}^{2}$. Likewise, $s_{k, 2}=1-2 s_{k-1,2}^{2}, \cdots, s_{k, L}=1-2 s_{k-1, L}^{2}$. Then Eq. (6) can be transformed into

$$
\begin{aligned}
g_{k}= & 1-2\left(p_{1} \times s_{k-1,1}^{2}+p_{2} \times s_{k-1,2}^{2}+\cdots\right. \\
& \left.+p_{L} \times s_{k-1, L}^{2}\right)
\end{aligned}
$$

From the above Eq. (7), we can notice that the proposed chaotic sequence based on weighted processing is a special case of the improved Logistic map chaotic sequences. Meanwhile, an excellent balance performance could be gained through adjusting weighted parameters flexibly.

\subsection{Analysis of correlation}

It is well known that conventional chaotic sequences based on the above maps have superiority in correlation characteristics including auto-correlation $(\mathrm{AC})$ and cross-correlation $(\mathrm{CC})$ because of high sensitivity to initial values. In chaotic SS communication systems, the capabilities of suppressing multipath and multiuser interference are characterized by the properties of $\mathrm{AC}$ and $\mathrm{CC}$, which are usually evaluated using $\mathrm{AC}$ function (ACF), $\mathrm{CC}$ function (CCF), AC sidelobe, and $\mathrm{CC}$ root-mean-square (RMS) value. However, it is uncertain whether the proposed sequence can provide the similar correlation performance as classical chaotic sequences or not. Next, the above four technical indexes of the proposed sequence will be tested.

Figures 3 and 4 show ACF and CCF of the above six chaotic sequences including the proposed sequence with relevant interval respectively. As shown in Figs. 3 and 4, the proposed sequence like other classical sequences has excellent properties of AC and CC. The variation curves of $\mathrm{AC}$ sidelobe and CC RMS value of six mentioned chaotic sequences with various code length are shown in Figs. 5 and 6 respectively. The two figures indicate that AC sidelobe and CC RMS value of six chaotic sequences are getting close to zero as the code length increases, which means the proposed sequence has excellent ability of suppressing multipath and multiuser interference as other classical sequences.

From trend of curves of the proposed sequence in Fig. 3 $\sim$ Fig. 6 , the property of correlation is qualitatively analyzed and the proposed sequence has excellent correlation performance as classical chaotic sequences. Besides, correlation property analysis also can be carried out from a statistical perspective. Table 1 shows sidelobe peak value of $\mathrm{AC}$ and $\mathrm{CC}$ for 6 chaotic codes in the condition of Doppler shift $1 \mathrm{KHz}$, where code length is 1023, each chaotic code chooses 10 sample sequences generated by

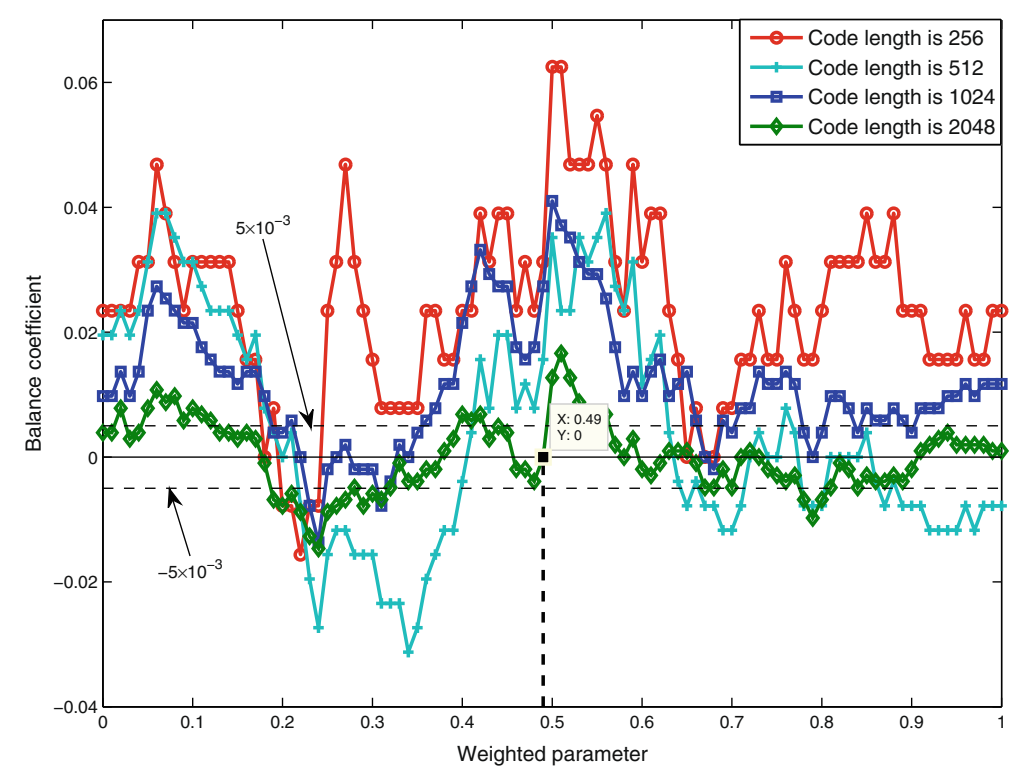

Fig. 2 Relationship between the balance coefficients of the proposed sequences and weighted parameters with various code length 

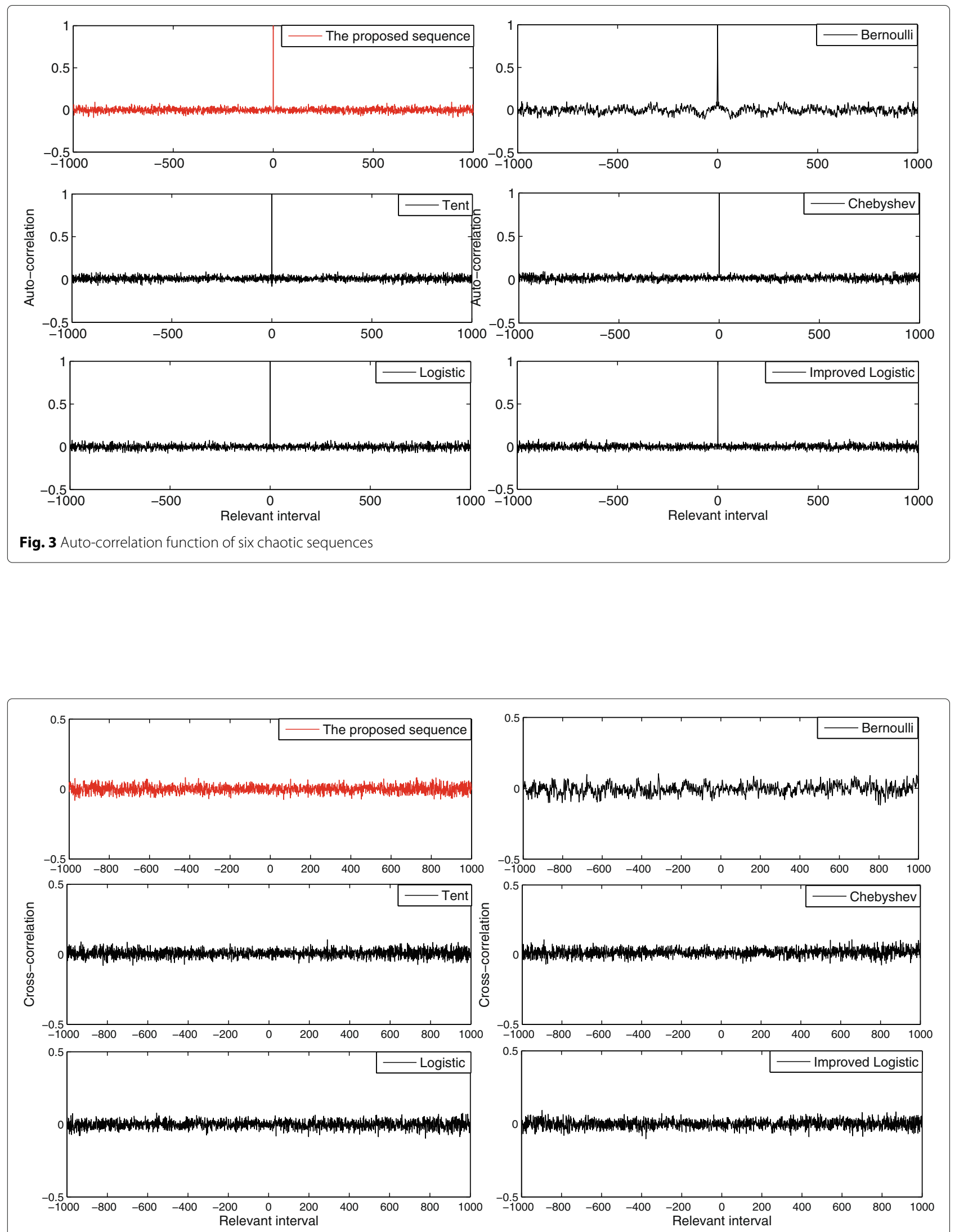

Fig. 4 Cross-correlation function of six chaotic sequences 

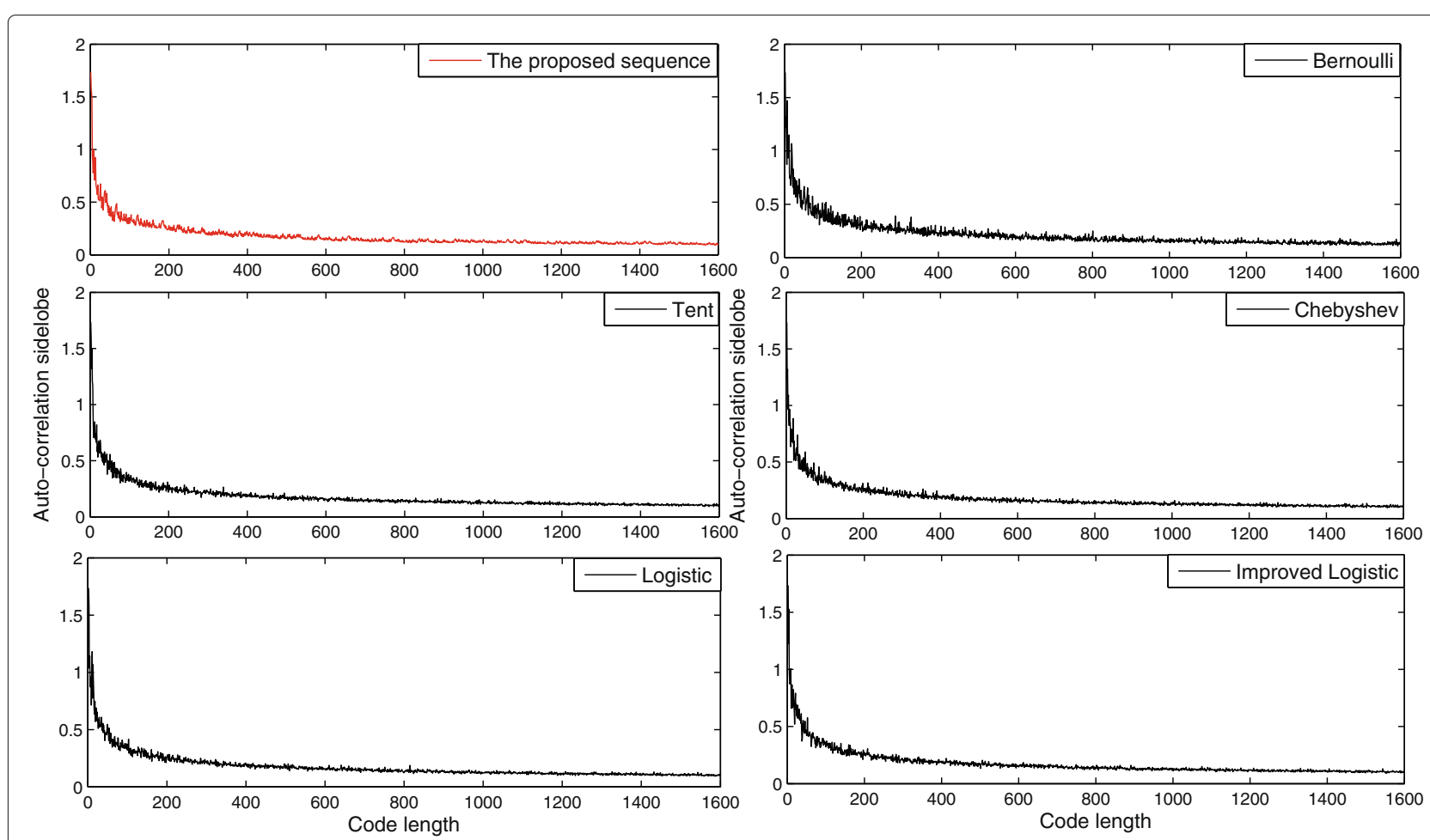

Fig. 5 Auto-correlation sidelobe of six chaotic sequences
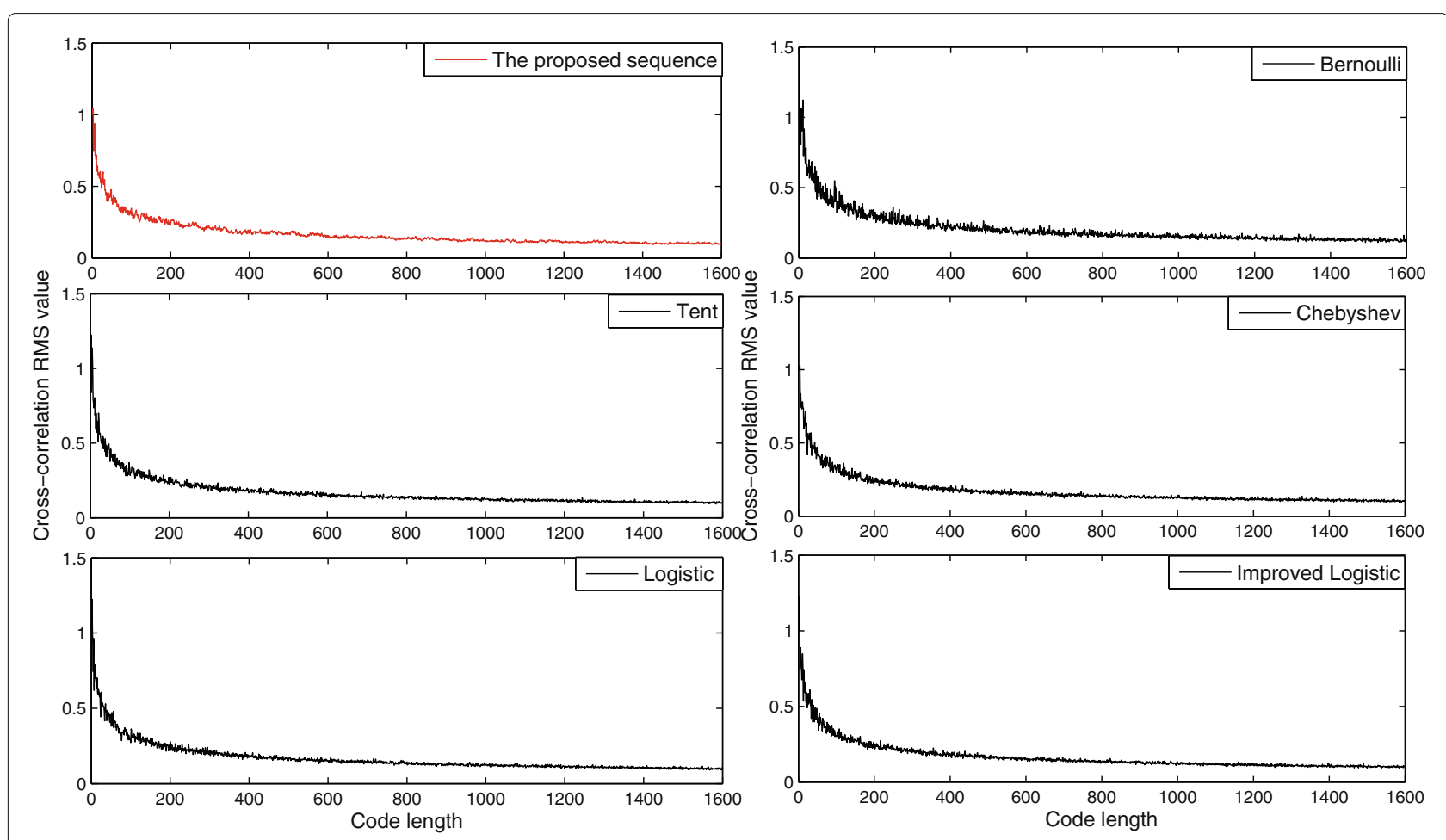

Fig. 6 RMS value of cross-correlation for six chaotic sequences 
Table 1 Sidelobe peak value of auto-correlation and cross-correlation for six chaotic codes (dB)

\begin{tabular}{lcccc}
\hline Ranging codes & MaxACFe & MaxACFo & MaxCCFe & MaxCCFo \\
\hline Chebyshev & -21.4636 & -21.6613 & -21.7129 & -20.7860 \\
Tent & -21.0266 & -20.8169 & -21.1114 & -20.8866 \\
Bernoulli & -21.7543 & -21.4409 & -21.0964 & -20.7382 \\
Logistic & -21.4893 & -21.5406 & -21.2028 & -20.8531 \\
Improved Logistic & -21.5507 & -21.3960 & -21.5682 & -21.2776 \\
The proposed code & -21.3509 & -21.2705 & -21.6414 & -21.2772 \\
\hline
\end{tabular}

random initial values, and MaxACFe, MaxACFo, MaxC$C F e$, and $M a x C C F o$ are denoted as even/odd AC sidelobe peak value and even/odd CC sidelobe peak value respectively. As shown in Table 1, the values of technical indexes among the above codes differ little quantitatively, which means the proposed code not only has good characteristic in balance but also maintains excellent correlation property as the conventional chaotic codes.

\subsection{Weil sequence}

Weil sequences [22] have excellent properties of pseudorandom and auto-correlation as well as relatively high linear complexity. They are based on Legendre sequences, which in turn are constructed from quadratic residues. The quadratic residue is defined as follows: if the greatest common divisor of $\alpha$ and $\beta$ is 1 , which is denoted as $(\alpha, \beta)=1$; if $\beta$ divided by $\left(x^{2}-\alpha\right)$ has a solution, namely $x^{2} \equiv \alpha(\bmod \beta)$, and $\alpha$ is called the quadratic residue of module $\beta$.

Weil sequences, the name has been chosen due to the dependence of correlation bound on Weil's classical bound of the magnitude of sums of Legendre symbols. The Legendre symbol $\left(\frac{x}{N}\right)$ is defined by:

$$
\frac{x}{N}=\left\{\begin{array}{l}
1 \text { if } x \text { is the quadratic residue of } N \\
0 \text { if } x \text { is not the quadratic residue of } N
\end{array}\right.
$$

where $N$ is the length of Legendre sequence.

$$
\operatorname{Leg}_{N}(i)=\left(\frac{i}{N}\right), \quad 1 \leq i \leq N-1
$$

with define: $\operatorname{Leg}_{N}(0)=0$. Weil sequence can be obtained by module two summation of Legendre sequence and its cyclic shift sequence, i.e.,

$\operatorname{Weil}_{N}^{t}(i)=\operatorname{Leg}_{N}(i) \oplus \operatorname{Leg}_{N}(i+t), \quad 1 \leq i \leq(N-1) / 2$

where $t$ is the shift value of the Legendre sequence.

\subsection{Random sequence}

The idea of random codes is to generate a family of codes that fulfills the properties of random noise as well as possible for a given code length [23]. Random sequence can be chosen from $2^{N R}$ possible permutation and combination of " 0 " and " 1 ," where $R$ is the number of the sequences, and $N$ is the length of a sequence. One possible implementation is the use of genetic algorithms that follow the principle of evolution and mutations, according to the Darwinist principle of the "survival of the fittest." The flowchart implemented for the optimization of the random codes can be seen in Fig. 7 .

\section{A weighted performance evaluation method based on the Welch bound}

The continuous improvements of the Compass signal structure have lead to the need of designing new spreading

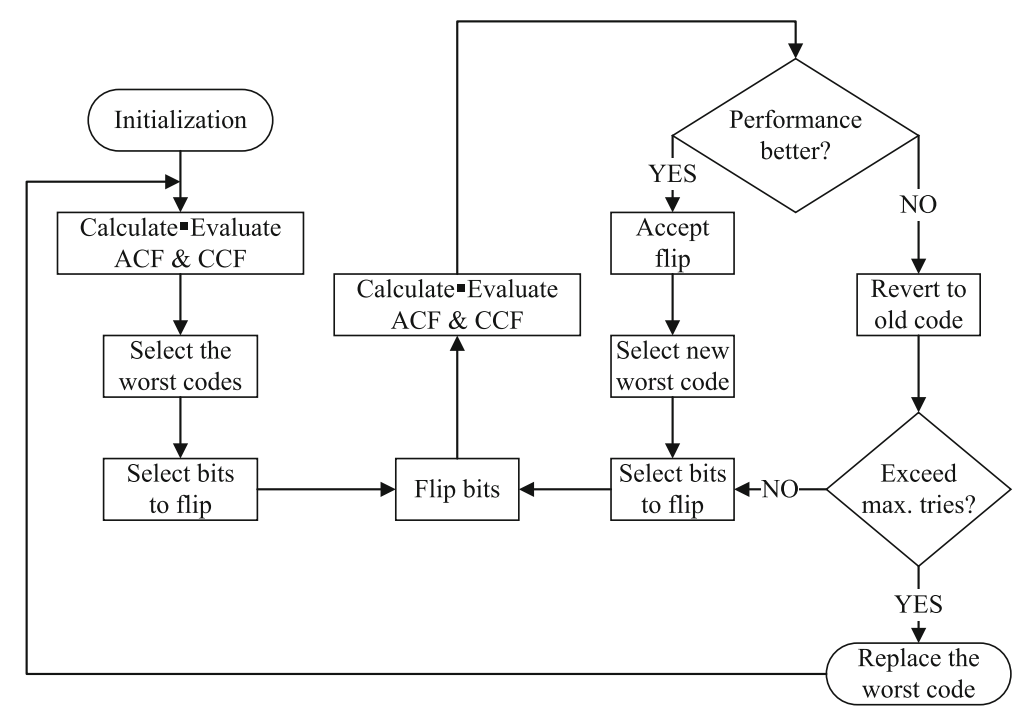

Fig. 7 The flowchart for optimization of Random sequence 
codes for the different open signals. In order to compare different candidates of code families, selection criteria have been set up and constantly strengthened over the last decade. These criteria model the impact of the spreading code characteristics and in particular of their auto- and cross-correlation functions onto the receiver performance. For this purpose, a comprehensive assessment method based on the Welch bound [24] including three main indexes has been proposed in [25], namely the performance in the acquisition mode, the tracking mode, and the robustness against interfering narrowband signals. Furthermore, these three indexes can be combined in a final figure or cost function in order to evaluate different candidate sets of codes. The corresponding weighted coefficients can be decided by the user groups, since the code impact varies for every type of application. The three performance indexes and cost function will be expounded in the following sections.

\subsection{The acquisition performance}

High values of the out-of-phase peak for the ACF or of the peaks for the CCF may lead to an increase of the probability of false acquisition. Mathematical models as presented in [26] are proposed to quantify this effect. One drawback is that they make some assumptions concerning the acquisition environment $\left(C / N_{0}\right.$ or Losses) or the receiver architecture (parallel or sequential acquisition). Consequently, it may be difficult to really distinguish the pure effect of correlation properties of a particular set of codes from the effects of these additional parameters. This is the reason why a simple criterion just depending on the code correlation properties has been designed to evaluate the effect of the code onto the acquisition performance. For this purpose, the Welch bound $\phi_{\min }$ has been introduced (see [24]). This bound is the theoretical minimum of the maximum value of auto- or cross-correlation functions that can be obtained for a given set of $M$ codes of the length $N$ and is defined as

$$
\phi_{\min }=N \sqrt{\frac{M-1}{M N-1}}
$$

Any correlation value exceeding $\phi_{\min }$ can be considered as a degradation risk for the acquisition performance. The higher the distance between these correlation values and the Welch bound is the higher the degradations have to be expected. The criterion built on the Welch bound is called Excess Welch Square Distance (EWSD). Two sub-criteria based on the EWSD have been distinguished in [25]. One $\left(E W S D^{M P}\right)$ quantifies the effects of multipath onto the acquisition performance and mainly uses the ACF. The other $\left(\mathrm{EWSD}^{\mathrm{CT}}\right)$ considers the effects of the non-desired signals onto the performances.

A typical scenario which justifies the introduction of the $\left(\mathrm{EWSD}^{\mathrm{MP}}\right)$ consists in considering a direct path signal undergoing a large attenuation, while other reflection components arrive at the receiver without suffering any degradation. This may be the case of indoor applications where multipath signals reflected at smooth surfaces (e.g., windows) are stronger than the direct path signal, that is attenuated through windows with a metal layer. During the acquisition process, the receiver may lock onto one of the sidelobes of the auto-correlation function. In order to avoid this, the Mean Excess Welch Squared Distance $\left(\mathrm{MEWSD}^{\mathrm{MP}}\right.$ ) criterion has been introduced as follows

$$
\operatorname{MEWSD}^{\mathrm{MP}}=\operatorname{mean}\left(\sum_{n f_{\text {offs }}} \frac{1}{2}(\underbrace{\underbrace{\sum_{l=1}^{N-1}\left(A C^{e}\left(l, f_{\text {offs }}\right)-\phi_{\text {off }}\right)>\phi_{\min }}_{l=1})^{2}}_{A C^{e}\left(l, f_{\text {ofs }}\right)>\phi_{\min }})\right)
$$

where $A C^{e}\left(l, f_{\text {offs }}\right)$ and $A C^{o}\left(l, f_{\text {offs }}\right)$ are respectively the even and odd ACF calculated for a relative delay $l$-times $T_{c}$ and for a Doppler frequency offset $f_{o f f s}$, and the sample number of Doppler frequency offset is $n_{f_{\text {offs }} \text {. In other }}$ words, the MEWSD ${ }^{\mathrm{MP}}$ represents the average value (over all possible delays and Doppler offsets) of the squared distances between the Welch bound and all even or odd ACF components exceeding this bound.

Equation (12) is evaluated for a single code. If all $M$ codes within a set have to be considered, then the Average Mean Excess Welch Square Distance (AMEWSD ${ }^{\mathrm{MP}}$ ) can be expressed as

$$
\operatorname{AMEWSD}^{\mathrm{MP}}=\frac{1}{M} \sum_{i=1}^{M} \operatorname{MEWSD}_{i}^{\mathrm{MP}}
$$

Another variant of the general EWSD criterion is determined by considering one more time that the direct path undergoes a large attenuation. Additionally, it is assumed that the navigation signals with the codes from other satellites are not attenuated. In this case, the cross-correlation peaks between the replica and all of these codes have to be taken into account. A similar expression as (12) can be deduced, by substituting the auto-correlation terms by the cross-correlation ones:

$\operatorname{MEWSD}_{i, j}^{C \mathrm{~T}}=\operatorname{mean}\left(\sum_{n f_{\text {offs }}} \frac{1}{2}(\underbrace{\underbrace{\sum_{l=0}^{N-1}\left(C C_{i, j}^{e}\left(l, f_{\text {offs }}\right)-\phi_{\min }\right)^{2}}_{l=0}+}_{C C_{i, j}^{e}\left(l, f_{\text {offs }}\right)>\phi_{\min }})\right)$ 
where $C C_{i, j}^{e}\left(l, f_{\text {offs }}\right)$ and $C C_{i, j}^{o}\left(l, f_{\text {offs }}\right)$ denote the even and odd CCF respectively for the sequences $i$ and $j$ when a relative delay is $l$-times $T_{c}$, and Doppler frequency offset is $f_{\text {offs }}$. Note that the value for $n_{f_{\text {offs }}}$ is identical to this used for the computation of the MESWD ${ }^{\mathrm{MP}}$.

Equation (14) provides the MEWSD ${ }^{\mathrm{CT}}$ between two different codes. If the whole set of $M$ codes has to be considered, then the averaged value is the figure of interest. The averaged MEWSD $^{\mathrm{CT}}$ (AMEWSD ${ }^{\mathrm{CT}}$ ) can be expressed as

$$
\operatorname{AMEWSD}^{\mathrm{CT}}=\frac{1}{M(M-1)} \sum_{i=1}^{M} \sum_{j=1}^{M} \operatorname{MEWSD}_{i, j}^{\mathrm{CT}}
$$

\subsection{The tracking performance}

For the acquisition mode, only the highest correlation peaks had to be considered to avoid any performance degradation. Now, in the tracking mode, all correlation components have to be taken into account, since they gather in one aggregate perturbation called average interference parameter. The corresponding criterion is called Merit Factor (MF). Similarly to the MEWSD, two cases, one for the multipath and another for the cross-talk, have been distinguished.

One of the main effects of multipath onto the tracking performance is modeled by the bias of the $\mathrm{S}$-curve. This effect is evaluated by the $\mathrm{MF}^{\mathrm{MP}}$ which can be expressed as follows

$$
\mathrm{MF}_{i}^{\mathrm{MP}}=\frac{1}{n_{f_{\text {offs }}}} \sum_{f_{\text {offs }}}\left\{\frac{1}{2}\left(\begin{array}{c}
\sum_{l=1,2, N-2, N-1}\left(A C_{i}^{e}\left(l, f_{\text {offs }}\right)\right)^{2}+ \\
\sum_{l=1,2, N-2, N-1}\left(A C_{i}^{o}\left(l, f_{\text {offs }}\right)\right)^{2}
\end{array}\right)\right\}
$$

The $\mathrm{MF}^{\mathrm{MP}}$ for a whole set of $M$ codes is then equal to the average of the corresponding $M$ values of $\mathrm{MF}^{\mathrm{MP}}$

$$
\mathrm{AMF}^{\mathrm{MP}}=\frac{1}{M} \sum_{i=1}^{M} \mathrm{MF}_{i}^{\mathrm{MP}}
$$

When considering the effect of all non-desired satellite signals onto the tracking performance, the following expression of MF has been tailored:

$$
\begin{aligned}
\mathrm{MF}_{i, j}^{\mathrm{CT}}=\frac{1}{n_{f_{\text {offs }}}} \sum_{f_{\text {offs }}} & \left(\frac{1}{2} \sum_{l=0}^{N-1}\left(C C_{i, j}^{e}\left(l, f_{\text {offs }}\right)\right)^{2}\right. \\
& \left.+\frac{1}{2} \sum_{l=0}^{N-1}\left(C C_{i, j}^{o}\left(l, f_{\text {offs }}\right)\right)^{2}\right)
\end{aligned}
$$

Hence the $\mathrm{MF}^{\mathrm{CT}}$ considers all possible CCF peaks (for any delay or Doppler shift) without any restriction regarding the bound of these Doppler offsets. As for the MEWSD criteria, the sampling interval for the $\mathrm{MF}^{\mathrm{CT}}$ is a fifth of the inverse of the code period. The $\mathrm{AMF}^{\mathrm{CT}}$ for a whole set of $M$ codes is equal to the average of the $M(M-1)$ elementary $\mathrm{MF}^{\mathrm{CT}}$ :

$$
\mathrm{AMF}^{\mathrm{CT}}=\frac{1}{M(M-1)} \sum_{i=1}^{M} \sum_{j=1}^{M} \mathrm{MF}_{i, j}^{\mathrm{CT}}
$$

\subsection{The robustness against interfering narrowband signals}

When the spreading code period becomes smaller, the power spectral density (PSD) for the corresponding navigation signals does not match the exact envelop of the pulse shape, as it would be the case for pure random codes (with infinite period), but rather shows peaks exceeding this envelop by several $\mathrm{dBs}$ (e.g., $8 \mathrm{~dB}$ for the GPS coarse/acquisition (C/A) codes). This property leads to an increase of the sensitivity against continuous wave signals or narrowband signals which are sent on these particular frequencies. Hence, a code showing good robustness against narrowband interfering signals must have as less peaks which exceed the ideal PSD for the pulse shape envelop as possible. Even for small data rates, these peaks do not represent any more points of vulnerability. Consequently, the Excess Line Weight (ELW) aims to reduce these peak exceedings.

ELW is a measure the robustness against narrowband signals. It is defined by

$$
\mathrm{ELW}=10 \lg (\frac{1}{n} \underbrace{\sum_{k=-n / 2}^{n / 2}\left(A_{k}-\sqrt{n}\right)^{2}}_{A_{k}>\sqrt{n}})
$$

where $A_{k}$ is the $k^{\text {th }}$ value of the discrete Fourier transform of the spreading codes, $c_{i}$. The ELW computes the averaged squared distances between the spectral line values and the value $\sqrt{n}$ which would be obtained for an ideal random code of infinite period. These averaged distances are only calculated for those spectral lines which exceed $\sqrt{n}$. The ELW for a whole set of $M$ codes is equal to the average of the $M$ elementary ELW:

$$
\mathrm{AELW}=\frac{1}{M} \sum_{i=1}^{M} \mathrm{ELW}_{i}
$$

\subsection{Sequence cost function}

Normally, performance analysis of ranging code cannot completely depend on a certain index. We should comprehensively evaluate the performance with several indexes, and each index is assigned a weighted value. Finally, comprehensive performance for ranging code is deter- 
mined by the sequence cost function $R_{i}$ through weighted fitting, i.e.,

$$
R_{i}=\sum_{j=1}^{5} \rho_{j} \frac{c v_{i, j}-\overline{c v_{j}}}{\overline{c v_{j}}}, \quad i=1,2, \cdots
$$

where $i$ is the index for different code sets, $j$ is the index for different criteria, $\rho_{j}$ is the weighted coefficient of criteria $j$ and $\rho_{1}+\rho_{2}+\cdots+\rho_{5}=1, c v_{i, j}$ is the criterion value of criteria $j$ and code set $i, \overline{c v_{j}}$ is the mean value of criteria $j$ over all different code sets, and $R_{i}$ is the result of performance in percentage or cost function. In this paper, we mainly consider five performance indexes, namely $\mathrm{AMEWSD}^{\mathrm{MP}}$, $\mathrm{AMEWSD}^{\mathrm{CT}}, \mathrm{AMF}^{\mathrm{MP}}, \mathrm{AMF}^{\mathrm{CT}}$, and AELW. The comprehensive performance including capture, tracking, and anti-jamming of the proposed sequences is evaluated in the following section compared to the traditional chaotic sequences, C/A, Gold, Weil, and Random sequences.

\section{The traditional types of interference}

There are various kinds of jamming modes. Active jamming can be divided into blanketing jamming and deceptive jamming, where the blanketing jamming can be divided into several modulation modes [27]. Three of the jamming modes, namely noise amplitude modulation (AM) jamming, noise frequency modulation (FM) jamming, and noise phase modulation (PM) jamming are discussed in this paper as follows.

\subsection{Noise AM jamming}

Noise AM jamming [28] makes use of additive white Gaussian noise (AWGN) to modulate the interference signal's amplitude and make it change with noise. That is

$$
n_{J 1}(t)=\left[a_{0}+a_{n}(t)\right] \cos \left(\omega_{j} t+\psi\right)
$$

where $n_{J 1}(t)$ is the signal of noise AM jamming, $a_{0}$ is original carrier amplitude, $a_{n}(t)$ is the bandlimited AWGN whose mean and variance are 0 and $\sigma^{2}$ respectively, $\omega_{j}$ is angular frequency of carrier, and initial phase $\psi$ is uniform distribution in $[0,2 \pi]$ and uncorrelated with $a_{n}(t)$. The received signal $y(t)$ is the sum of useful signal $s(t)$, interference signal $n_{J}(t)$, and thermal noise $n(t)$, i.e.,

$$
y(t)=s(t)+n_{J 1}(t)+n(t)
$$

\subsection{Noise FM jamming}

Noise FM jamming [29] makes use of AWGN to modulate the interference signal's frequency and make it change with noise. That is

$$
n_{J 2}(t)=U_{j} \cos \left(\omega_{j} t+2 \pi K_{F M} \int_{0}^{t} u_{n}(\tau) d \tau+\psi\right)
$$

where $n_{J 2}(t)$ is the signal of noise FM jamming, $U_{j}$ is the amplitude of noise FM jamming, $K_{\mathrm{FM}}$ is frequency modulating factor, which indicates the frequency change caused by unit noise signal, $u_{n}(t)$ is the bandlimited AWGN whose mean and variance are 0 and $\sigma^{2}$ respectively, $\omega_{j}$ is
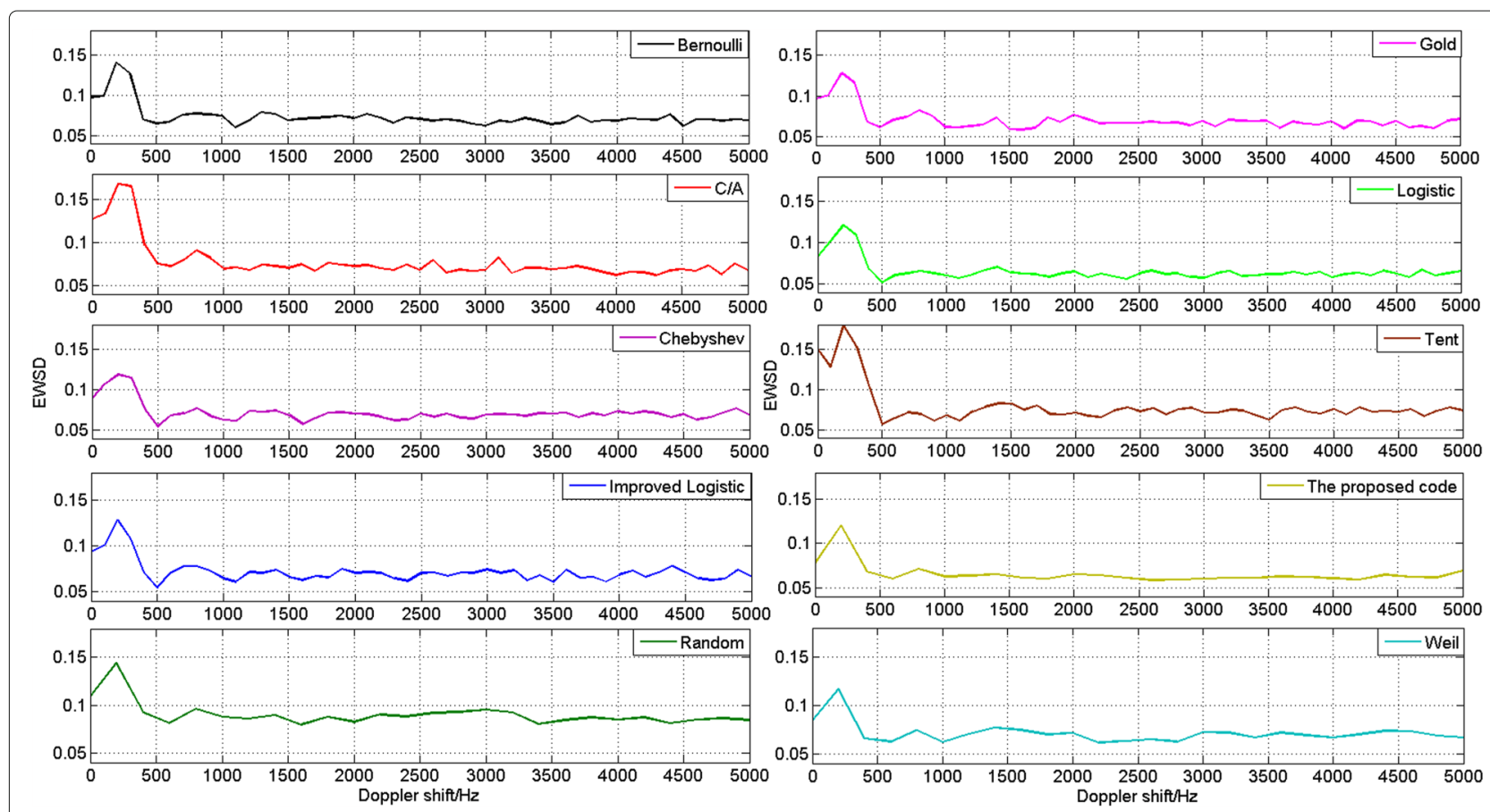

Fig. 8 Relation curves between Doppler shift and EWSD of 10 different sequences

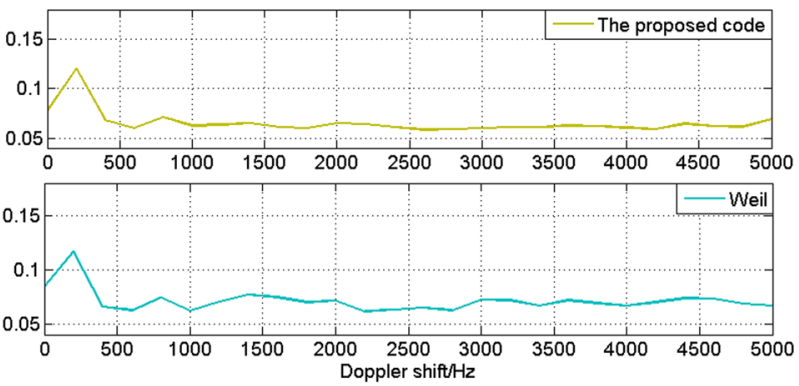


Table 2 The comparison of performance indexes for various ranging codes

\begin{tabular}{|c|c|c|c|c|c|}
\hline Ranging codes & AMEWSD $^{M P}$ & AMEWSD $^{C T}$ & $\mathrm{AMF}^{\mathrm{MP}}$ & $\mathrm{AMF}^{\mathrm{CT}}$ & AELW \\
\hline $\mathrm{C} / \mathrm{A}$ & 0.0757 & 0.0746 & $6.3700 \times 10^{-5}$ & 1.0017 & 18.0553 \\
\hline Gold & 0.0715 & 0.0706 & $1.7903 \times 10^{-4}$ & 1.0001 & 17.5296 \\
\hline Logistic & 0.0640 & 0.0649 & $1.7443 \times 10^{-4}$ & 1.0014 & 17.2405 \\
\hline Improved Logistic & 0.0712 & 0.0718 & $2.2310 \times 10^{-4}$ & 1.0028 & 17.2680 \\
\hline Chebyshev & 0.0701 & 0.0719 & $1.1625 \times 10^{-4}$ & 1.0026 & 17.2296 \\
\hline Bernoulli & 0.0729 & 0.0728 & $8.4607 \times 10^{-5}$ & 1.0044 & 17.2903 \\
\hline Tent & 0.0758 & 0.0740 & $1.7949 \times 10^{-4}$ & 1.0185 & 17.4540 \\
\hline Weil & 0.0703 & 0.0710 & $1.1586 \times 10^{-4}$ & 1.0053 & 17.4074 \\
\hline Random & 0.0937 & 0.0927 & $1.9021 \times 10^{-4}$ & 1.0047 & 18.3198 \\
\hline The proposed code & 0.0640 & 0.0641 & $7.5566 \times 10^{-5}$ & 0.9992 & 17.2249 \\
\hline
\end{tabular}

angular frequency of carrier, and initial phase $\psi$ is uniform distribution in $[0,2 \pi]$ and uncorrelated with $u_{n}(t)$.

\subsection{Noise PM jamming}

Noise PM jamming [30] makes use of noise to modulate the interference signal's phase and make it change with noise. That is

$$
n_{J 3}(t)=U_{j} \cos \left(\omega_{j} t+K_{P M} u_{n}(t)+\psi\right)
$$

where $n_{J 3}(t)$ is the signal of noise PM jamming, $U_{j}$ is the amplitude of noise PM jamming, $K_{P M}$ is phase modulating factor which indicates the phase change caused by unit noise signal, $u_{n}(t)$ is the bandlimited AWGN whose mean and variance are 0 and $\sigma^{2}$ respectively, $\omega_{j}$ is angular frequency of carrier, and initial phase $\psi$ is uniform distribution in $[0,2 \pi]$ and uncorrelated with $u_{n}(t)$.

\section{Simulation results and analysis}

The relation curves between Doppler shift and EWSD of 10 kinds of sequences including classical chaotic sequences, C/A, Gold, Weil, and Random as well as the proposed sequence are shown in Fig. 8, where the length of each sequence is 1023, and code rate is $1.023 \mathrm{Mbit} / \mathrm{s}$ and each ranging code randomly chooses 10 sequences as testing samples. As seen from the Fig. 8, EWSD has a large value when Doppler shift is small, and changing trend of EWSD is increasing at first then decreasing rapidly when Doppler shift is varied from 0 to $1000 \mathrm{~Hz}$. EWSD descends with the increasing of Doppler shift and tends to be stable when Doppler shift is more than $1000 \mathrm{~Hz}$.

On the one hand, we expect to use plenty of sampling points to describe the changing details of EWSD. On the other hand, too many sampling points will increase the complexity of hardware implementation, so the sampling number of Doppler shift $n_{f_{\text {offs }}}$ for computing EWSD needs to be comprehensively considered. In this paper, the range of Doppler shift is set from 0 to $6800 \mathrm{~Hz}$, and sampling interval is $200 \mathrm{~Hz}$, and $n_{f_{\text {offs }}}$ is equal to 35 . The comparison of performance indexes based on the Welch bound evaluation criteria for various sequences is listed in Table 2. In order to completely evaluate the performance of the above

Table 3 The comparison of performance indexes for various ranging codes through weighting (\%)

\begin{tabular}{|c|c|c|c|c|c|}
\hline Ranging codes & AMEWSD $^{M P}$ & AMEWSD $^{C T}$ & $\mathrm{AMF}^{\mathrm{MP}}$ & $\mathrm{AMF}^{\mathrm{CT}}$ & AELW \\
\hline$C / A$ & 0.7625 & 0.4833 & -10.9145 & -0.0472 & 0.6323 \\
\hline Gold & -0.3895 & -0.6150 & 5.5349 & -0.0791 & 0.0316 \\
\hline Logistic & -2.4465 & -2.1801 & 4.8788 & -0.0532 & -0.2988 \\
\hline Improved Logistic & -0.4717 & -0.2856 & 11.8205 & -0.0253 & -0.2673 \\
\hline Chebyshev & -0.7735 & -0.2581 & -3.4194 & -0.0293 & -0.3112 \\
\hline Bernoulli & -0.0055 & -0.0110 & -7.9326 & 0.0066 & -0.2418 \\
\hline Tent & 0.7899 & 0.3185 & 5.6005 & 0.2874 & -0.0548 \\
\hline Weil & -0.7186 & -0.5052 & -3.4750 & 0.0245 & -0.1080 \\
\hline Random & 5.6994 & 5.4530 & 6.1295 & 0.0125 & 0.9346 \\
\hline The proposed code & -2.4465 & -2.3998 & -9.2221 & -0.0970 & -0.3166 \\
\hline
\end{tabular}


sequences, sequence cost function denoted by Eq. (22) as a tool is introduced to assess comprehensive performance. The weighted coefficient $\rho_{j}(j=1,2, \cdots, 5)$ is set at 0.2 , which refers to the setting of weighted value in the course of seeking optimum ranging codes for Galileo system [25]. The performance indexes of the above sequences through weighed processing are shown in Table 3.

From both of Tables 2 and 3, we can note that the proposed sequence has the minimum value the same as the Logistic sequence in $\mathrm{AMEWSD}^{\mathrm{MP}}$ and has the minimum value in AMEWSD ${ }^{C T}$. Comprehensively, the proposed sequence has better acquisition performance than other sequences. In terms of $\mathrm{AMF}^{\mathrm{MP}}$, the proposed sequence is smaller than other sequences except $\mathrm{C} / \mathrm{A}$ sequence, which indicates the proposed sequence has better tracking performance against multipath effect than other sequences, inferior to $\mathrm{C} / \mathrm{A}$. In addition, the proposed sequence has the minimum value in $\mathrm{AMF}^{\mathrm{CT}}$ and AELW, which means the proposed sequence has the best tracking performance against other satellites' signal jamming and optimal performance of anti-narrowband interference.

For the sake of testing performance of the proposed sequence under three aforementioned interference, a system composed by $(2,1,3)$ convolutional code, binary phase shift keying (BPSK), and a few of direct spread spectrum sequences is established. Figures 9, 10, and 11 show the bit error rate (BER) performance of the system with jamming-to-signal ratio (JSR) under noise AM, FM, and PM jamming over AWGN channel respectively. In these simulation, the length of information bits is 200 , spread spectrum code length is 1023 , the number of frames is 5000, both of modulating factor in noise FM and $\mathrm{PM}$ jamming are 0.8 , and signal-to-noise ratio (SNR) is $5 \mathrm{~dB}$. Figures 9, 10, and 11 show that all sequences' BER performance is improved with the decreasing of JSR, and

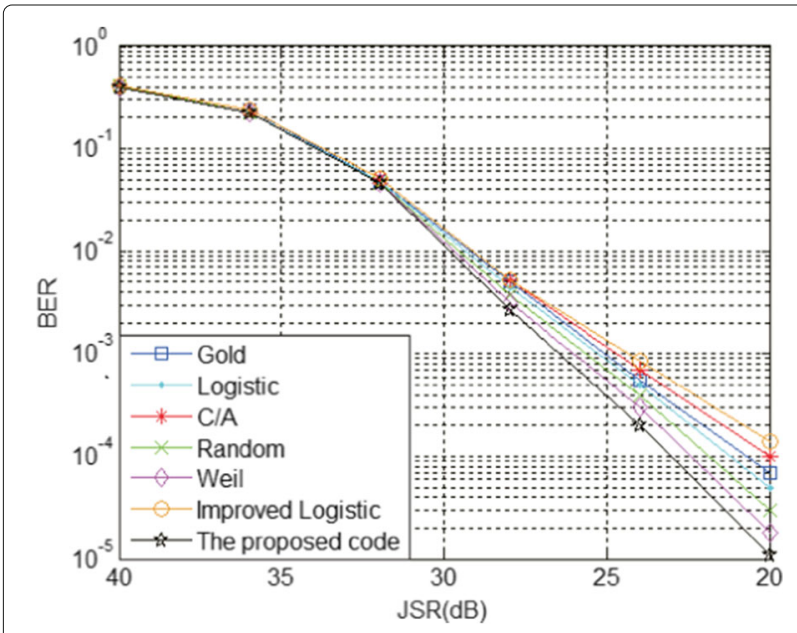

Fig. 9 BER curve of ranging codes with JSR under noise AM jamming

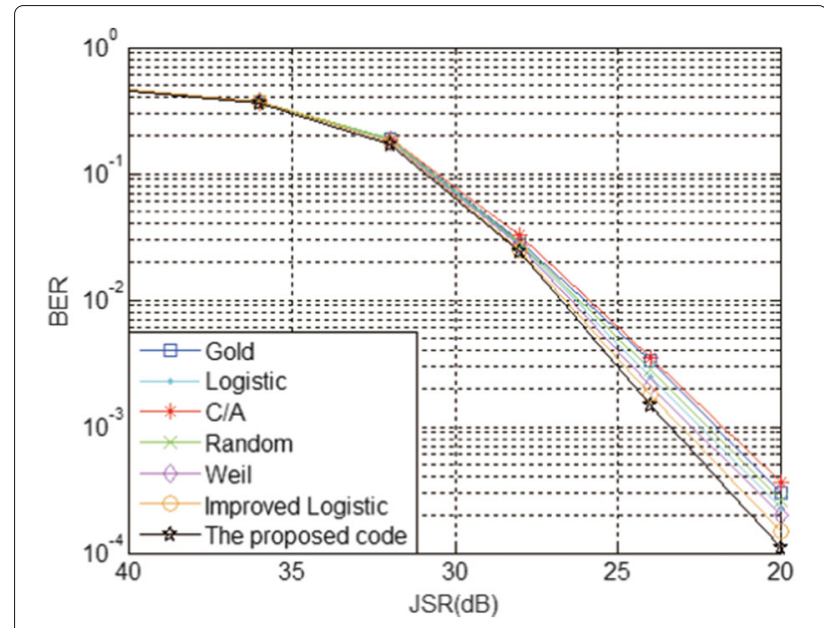

Fig. 10 BER curve of ranging codes with JSR under noise FM jamming

the proposed sequence has the best BER performance in all sequences under three typical jamming when JSR is more than $32 \mathrm{~dB}$.

\section{Conclusions}

In view of the disadvantage of conventional chaotic sequences in terms of balance, a novel ranging code is presented, which is constructed by some improved Logistic map chaotic sequences using different initial values through weighted optimization, summation, and quantization. From construction process, we can see that the proposed codes are not only related to initial values but also related to weighted parameters, and it means that the ability of anti-decipher is enhanced. A weighted performance assessment method based on the Welch bound is introduced to evaluate the proposed ranging codes, C/A, Gold, Weil, Random, and the classical chaotic codes. A lot of simulation results in AWGN channel show that

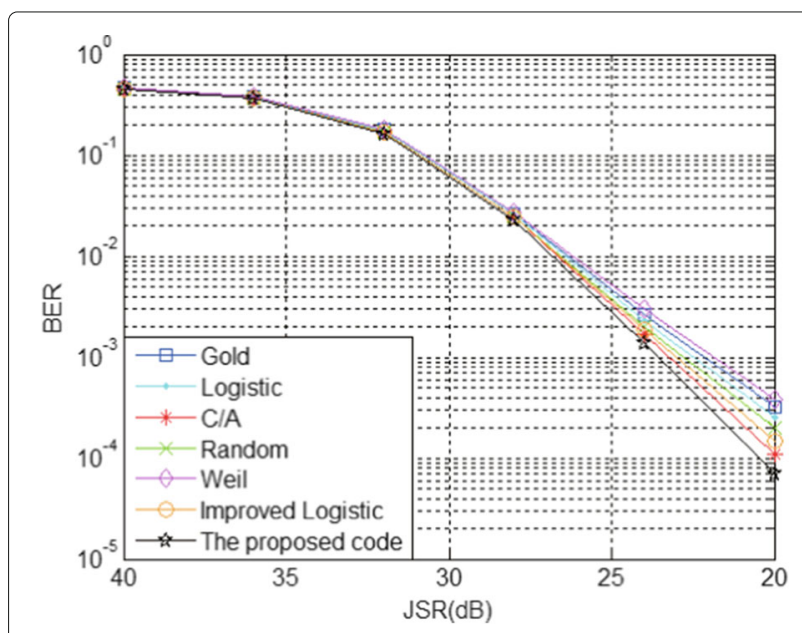

Fig. 11 BER curve of ranging codes with JSR under noise PM jamming 
the proposed ranging code cannot only have the best performance in acquisition and anti-narrowband interference among the above ranging codes and keep excellent tracking performance as $\mathrm{C} / \mathrm{A}$ code but also greatly improve the balance performance and strengthen reliability and security.

\begin{abstract}
Abbreviations
AC: Auto-correlation; ACF: Auto-correlation function; AELW: Average excess line weight; AM: Amplitude modulation; AMEWSD: Average mean excess Welch square distance; AMF: Average merit factor; AWGN: Additive white gaussian noise; BER: Bit error rate; BPSK: Binary phase shift keying; C/A: Coarse/Acquisition; CC: Cross-correlation; CCF: Cross-correlation function; ELW: Excess line weight; EWSD: Excess Welch square distance; FM: Frequency modulation; GLONASS: Russian global navigation satellite system; GNSS: Global navigation satellite system; GPS: Global positioning system; IRNSS: Indian regional navigation satellite system; JSR: Jamming-to-signal ratio; LFSR: Linear feedback shift register; MEWSD: Mean excess Welch squared distance; MF: Merit factor; PM: Phase modulation; PSD: Power spectral density; QZSS: Quasi-Zenith satellite system; RMS: Root-mean-square; SNR: Signal-to-noise ratio; SS: Spread spectrum
\end{abstract}

\section{Acknowledgements}

This paper is supported by the Open Research Fund of State Key Laboratory of Space-Ground Integrated Information Technology (Grant No.

2015_SGIIT_KFJJ_DH_03), the Open Research Fund of State Key Laboratory of Tianjin Key Laboratory of Intelligent Information Processing in Remote Sensing (Grant No. 2016-ZW-KFJJ-01),the National Natural Science Foundation of China (Grant No. 61403093), the Science Foundation of Heilongjiang Province of China for Returned Scholars (Grant No. LC2013C22), the Assisted Project by Heilongjiang Province of China Postdoctoral Funds for Scientific Research Initiation (Grant No. LBH-Q14048), and the Fundamental Research Funds for the Central universities (Grant No. HEUCF0817).

\section{Competing interests}

The authors declare that they have no competing interests.

\section{Publisher's Note}

Springer Nature remains neutral with regard to jurisdictional claims in published maps and institutional affiliations.

\section{Author details}

${ }^{1}$ State Key Laboratory of Space-Ground Integrated Information Technology, 100086 Beijing, China. ${ }^{2}$ College of Information and Communication Engineering, Harbin Engineering University, 150001 Harbin, China.

Received: 1 October 2016 Accepted: 13 March 2017

Published online: 27 March 2017

\section{References}

1. CX Yang, XC Lu, L Wang, Performance analysis for ranging codes of satellite navigation system. J, Time Freq. 36(6), 173-180 (2013)

2. J Du, J Guo, XC Lu, X Wang, L Yang, J Ruan, in IEEE International Frequency Control Symposium (FCS). The optimum selection method and performance analysis for Weil code of satellite navigation system, (Taipei, 2014), pp. 1-4

3. X Liu, M Liang, Y Morton, P Closas, T Zhang, Z Hong, Performance evaluation of MSK and OFDM modulations for future GNSS signals. GPS Solution. 18, 163-175 (2014)

4. R Xue, QM Cao, Q Wei, A flexible modulation scheme design for C-band GNSS signals. Math. Problems Eng. 2015, 1-16 (2015)

5. MH Liu, XQ Zhan, W Li, M Chen, A compatibility analysis between GNSS and radio astronomy/microwave landing system in $C$ band. J. Aeronaut. Astronautics Aviat. 46(2), 102-107 (2014)

6. W Wang, CC LV, X li, Assessment models and rules of GNSS interoperability. Adv. Mater. Res. 846, 808-811 (2014)

7. JJ Zhang, M Xue, J Xie, Research on assessment method of intrasystem and intersystem of the global navigation satellite system. Sci. China Technol. Sci. 58(10), 1672-1681 (2015)
8. W Liu, Y Hu, A method for determination of GNSS radio frequency compatibility threshold and its assessment. IEICE Trans. Commun. 97(5), 1103-1111 (2014)

9. TE Humphreys, Detection strategy for cryptographic GNSS anti-spoofing. IEEE Trans. Aerospace Electron. Syst. 49(2), 1073-1090 (2013)

10. RZ He, JX Cui, in AASRI Winter International Conference on Engineering and Technology (AASRI-WIET). Research of pseudo random noise code and new code construction, (Saipan, 2013), pp. 147-150

11. B Wang, GJ Wen, K Xie, On the synchronization of a hyperchaotic system based on adaptive method. Phys. Lett. A. 372(17), 3015-3020 (2008)

12. BWang, GJ Wen, On the synchronization of uncertain master-slave chaotic systems with disturbance. Chaos Solitons Fractals. 41(1), 145-151 (2009)

13. T Kohda, A Tsuneda, T Sakae, in IEEE Second International Symposium on Spread Spectrum Techniques and Applications. Chaotic binary sequences by Chebyshev maps and their correlation properties, (Yokohama, 1992), pp. 63-66

14. TKohda, A Tsuneda, Pseudonoise sequences by chaotic nonlinear maps and their correlation properties. IEICE Trans. Commun. 76(8), 855-862 (1993)

15. JM Lipton, KP Dabke, Spread spectrum communications based on chaotic systems. Int. J. Bifurcation Chaos. 6(12a), 2361-2374 (1996)

16. T Kohda, A Tsuneda, Statistics of chaotic binary sequences. IEEE Trans. Inform. Theory. 43(1), 104-112 (1997)

17. C Fatima, D Ali, New chaotic binary sequences with good correlation property using logistic maps. IOSR J. Electron. Commun. Eng. 5(3), 59-64 (2013)

18. U Parlitz, S Ergezinger, Robust communication based on chaotic spreading sequences. Phys. Lett. A. 188(2), 146-150 (1994)

19. H Wang, JD Hu, The improved Logistic-map chaotic spread spectrum sequences. J. China Inst. Commun. 18(8), 71-77 (1997)

20. Y He, S Yang, Q Xu, Short-term cascaded hydroelectric system scheduling based on chaotic particle swarm optimization using improved logistic map. Commun. Nonlinear Sci. Numerical Simul. 18(8), 1746-1756 (2013)

21. YH Yu, SZ Ma, WD Liu, Performance of balance based on Chebyshev 2-phase chaotic spreading sequence. J. Jilin Univ. 22(5), 228-231 (2004)

22. JJ Rushanan, in IEEE International Symposium on Information Theory. Weil sequences: a family of binary sequences with good correlation properties, (Seattle, 2006), pp. 1648-1652

23. S Wallner, JA Avila-Rodriguez, GW Hein, JJ Rushanan, in ION GNSS 20th international technical meeting of the satellite division. Galileo E1 OS and GPS L1C pseudo random noise codes-requirements, generation, optimization and comparison, (Fort Worth, 2007), pp. 25-28

24. L Welch, Lower bounds on the maximum cross correlation of signals. IEEE Trans. Inform. Theory. IT-20(3), 397-399 (1974)

25. F Soualle, M Soellner, S Wallner, JA Avila-Rodriguez, in The European navigation conference GNSS. Spreading code selection criteria for the future GNSS Galileo, (Munich, 2005), pp. 19-22

26. WR Braun, PN acquisition and tracking performance in DS/CDMA systems with symbol-length spreading sequences. IEEE Trans. Commun. 45(12), 1595-1601 (1997)

27. XF Wu, DH Dai, XS Wang, in IET International Conference on Radar Systems. Study on SAR jamming measures, (Edinburgh, 2007), pp. 1-5

28. YC Tu, HC Zhao, XG Zhou, in IEEE International Symposium on Microwave, Antenna, Propagation and EMC Technologies for Wireless Communications. Performance analysis of anti-aiming noise AM jamming for pseudo-random code phase modulation and PAM combined fuze, (Hangzhou, 2007), pp. 1171-1174

29. XG Zhou, HC Zhao, YC Tu, in IEEE International Symposium on Microwave, Antenna, Propagation and EMC Technologies for Wireless Communications. Performance analysis of anti-noise FM jamming of pseudo-random code binary phase modulation fuze based on doppler effect, (Hangzhou, 2007), pp. 1424-1427

30. DG Xie, J Li, C Wang, Q Liu, S Ye, in IEEE International Symposium on Microwave, Antenna, Propagation and EMC Technologies for Wireless Communications. Analysis and simulation of typical mode of jamming on data link communication system, (Chengdu, 2013), pp. 530-533 